\title{
Health-related Quality of Life Outcomes of Adalimumab for Patients With Rheumatoid Arthritis in Korea
}

\author{
Myeung-su Lee, M.D., Ph.D. ${ }^{1}$, Chang Hoon Lee, M.D., Ph.D. ${ }^{1}$, Hye Soon Lee, M.D., Ph.D. ${ }^{2}$, \\ Yoon-Kyoung Sung, M.D., Ph.D. ${ }^{3}$, Jung Ran Choi, M.D., Ph.D. ${ }^{4}$, Kyungsu Park, M.D., Ph.D. ${ }^{5}$, \\ Mi-Kyoung Lim, M.D., Ph.D. ${ }^{6}$, Byoong Yong Choi, M.D. ${ }^{7}$, Hyoun-Ah Kim, M.D., Ph.D. ${ }^{8}$, \\ Seung Won Choi, M.D., Ph.D. ${ }^{9}$, Yusun Lee, M.D. ${ }^{10}$, Wan-Hee Yoo, M.D., Ph.D. ${ }^{11}$ \\ ${ }^{1}$ Department of Internal Medicine, Wonkwang University Hospital, Iksan, ${ }^{2}$ Department of Internal Medicine, Hanyang University Guri Hospital, \\ Hanyang University School of Medicine, Guri, ${ }^{3}$ Department of Rheumatology, Hanyang University Hospital for Rheumatic Diseases, Seoul, \\ ${ }^{4}$ Department of Internal Medicine, Pohang St. Mary's Hospital, Pohang, ${ }^{5}$ Department of Internal Medicine, St. Vincent's Hospital, College of \\ Medicine, The Catholic University of Korea, Suwon, ${ }^{6}$ Department of Internal Medicine, Eulji University Hospital, Eulji University School of \\ Medicine, Daejeon, ${ }^{7}$ Department of Internal Medicine, Seoul Medical Center, Seoul, ${ }^{8}$ Department of Rheumatology, Ajou University School of \\ Medicine, Suwon, ${ }^{\prime}$ Department of Internal Medicine, Ulsan University Hospital, University of Ulsan College of Medicine, Ulsan, ${ }^{10}$ AbbVie Ltd, \\ Seoul, ${ }^{11}$ Department of Internal Medicine, Jeonbuk National University Medical School, Research Institute of Clinical Medicine of Jeonbuk \\ National University-Biomedical, Research Institute of Jeonbuk National University Hospital, Jeonju, Korea
}

Objective. Rheumatoid arthritis (RA) is a chronic, progressive, autoimmune disorder that impairs patients' overall health-related quality of life (HRQOL). In this study, we evaluated the effect of adalimumab in Korean patients with active RA on HRQOL. Methods. Patients included in the study had moderate to severe active RA that did not respond to conventional drugs with a Disease Activity Score of 28 joints $>3.2$ and were biologics-naïve. All patients received adalimumab 40 mg subcutaneously every other week and were followed for 24 weeks. The primary endpoint was the change in baseline Health Assessment Questionnaire Disability Index (HAQ-DI) score at week 24. Secondary endpoints were changes in the EuroQol 5-dimension 3-Level (EQ-5D-3L) baseline score and Short Form 36-Item Health Survey (SF-36) domain scores at weeks 12 and 24 and change in baseline HAQ-DI score at week 12. Results. In total, 91 Korean patients were included. Ninety-three percent of patients were in high disease activity with a baseline mean DAS28 value of 6.1 within all patients. The mean change from baseline in HAQ-DI scores were -0.46 at week 12 and $~ 0.67$ at week $24(p<0.0001)$. Additionally, EQ-5D-3L score at weeks 12 and 24 had significantly improved $(p<0.0001)$ compared to baseline. SF-36 at weeks 12 and 24 had significantly improved $(p<0.0001, p=0.0001)$ compared to baseline. Conclusion. Treatment with adalimumab resulted in significant improvement in HAQ-DI, EQ-5D-3L, and SF-36 scores at 12 and 24 weeks in Korean RA patient. (J Rheum Dis 2021;28:68-75)

Key Words. Adalimumab, Patients, Quality of life, Rheumatoid arthritis, Antirheumatic agents

\section{INTRODUCTION}

Rheumatoid arthritis (RA) is a systemic disorder with a prevalence of approximately $0.5 \% \sim 1 \%$ worldwide that causes joint destruction due to bone erosion [1]. More than half of patients with RA have one or more comorbidities over their lifetimes [2]. Cardiovascular disease, res- piratory disease, and depression are the most common, with a high probability of dyslipidemia and infectious diseases as well [3]. Recent studies in Korea reported a significant increase in mortality and disability in RA patients compared to the general population [4]. Patients with RA not only complain of discomfort in daily life due to reduced health-related quality of life (HRQOL) in terms of

Received : June 29, 2020, Revised : (1st) November 3, 2020, (2nd) November 26, 2020, Accepted : November 26,2020

Corresponding to : Wan-Hee Yoo id http://orcid.org/0000-0002-8430-7629

Department of Internal Medicine, Jeonbuk National University Medical School, Research Institute of Clinical Medicine of Jeonbuk National University-Biomedical, Research Institute of Jeonbuk National University Hospital, 20 Geonji-ro, Deokjin-gu, Jeonju 54907, Korea. E-mail : ywhim@jbnu.ac.kr 
pain, fatigue, and functional impairment caused by disease but also experience decreased productivity in vocational practice. In terms of holistic patient treatment, it is important to enable everyday activities considering psychological state and social roles in addition to preventing pain and joint deformation $[5,6]$. Therefore, early and active RA treatment to maintain patient function is an important social and economic issue. An accurate assessment of patient HRQOL is an important criterion for judging the therapeutic effect of RA on the patient's side and is important in evaluating drug efficacy in clinical studies. The most commonly used indicators of HRQOL worldwide are the Health Assessment Questionnaire Disability Index (HAQ-DI) [7], 36-item Short Form questionnaire (SF-36) [8,9], and EuroQol 5-dimension 3-Level (EQ-5D-3L) [10]. These are indicators that reflect the actual treatment effect in terms of reflecting the patients' lives in the actual environment. The advent of biological disease- modifying antirheumatic drugs (DMARDs) have revolutionized the management of RA since they can rapidly decrease disease activity, although the cost of treatment with biological DMARDs is higher than that of traditional DMARDs [11]. Adalimumab, a human tumor necrosis factor- $\alpha$ (TNF $\alpha$ ) monoclonal antibody, was found effective in the treatment of RA in adults [12]. It received United States Food and Drug Administration approval in 2002 for the treatment of RA as monotherapy or in combination with methotrexate or other DMARDs [13]. In Korea, adalimumab is reimbursed for both moderate and severe RA patients and it is the most widely used TNF inhibitory drug for treatment of rheumatoid arthritis patients in Korea to date.

The purpose of this study is to investigate the effect of adalimumab on patient HRQOL and labor productivity in patients with moderate to severe RA. This is the first attempt in South Korea to investigate the effect of adalimumab on HRQOL to maintain individual health and happiness while managing patients with RA.

\section{MATERIALS AND METHODS}

\section{Patients and study design}

This prospective observational non-interventional study assessed the effect of adalimumab on HRQOL and work productivity in patients with RA in Korea in clinical practice. Across 9 sites, 91 patients aged 18 years or older who met the revised 1987 American College of Rheumatology/ European League against Rheumatism 2010 classi- fication criteria were enrolled. Patients included in the study had moderate to severe active RA that did not respond to conventional drugs with a Disease Activity Score of 28 joints (DAS28) of erythrocyte sedimentation rate or C-reactive protein level $>3.2$ and were biologics-naïve. Patients who were pregnant or breast feeding at the time of enrollment or planned to become pregnant in the next 24 weeks or who participated in any RA-related clinical trial at the time of enrollment, at baseline or at any point during the past 24 weeks prior to baseline, or who were not able to accurately report their QoL or prior resource utilization, or who were not able to adhere to adalimumab therapy over 24 weeks from the clinician's view were excluded. Patients' comorbidities including myocardial infarction, congestive heart failure, peripheral vascular disease, cerebrovascular disease, dementia, chronic lung disease, connective tissue disease, ulcer disease, liver disease, diabetes, unilateral paralysis, moderate to severe kidney disease, tumors, leukemia, lymphoma, and AIDS were collected based on the patient's medical records at baseline. A biweekly dose of adalimumab $40 \mathrm{mg}$ was administered to patients and assessments using the HAQ-DI, SF-36, EQ-5D-3L, and Work Productivity and Activity Impairment (WPAI) were performed at baseline, Week 12, and Week 24. Their clinical and sociodemographic data were widely collected through interviews, self-administered questionnaires, and clinical examinations. At study initiation (baseline), the patients completed the study questionnaires in paper form. At all follow up visits (week 12 and 24), the patient completed the study questionnaires while waiting for their appointment at their clinic, approximately 30 minutes before they visit their physicians. It is important that they complete the questionnaires prior to speaking with their clinician during the visit, as the clinician feedback might impact patient perceptions measured by the patient reported outcome (PRO) instruments. Adverse event (AE) was collected at baseline, week 12, week 24, early termination or drop out, and a follow-up phone call approximately 70 days after the last administration of adalimumab. An AE was defined as any untoward medical occurrence in a patient, which does not necessarily have a causal relationship with their treatment. If an adverse event meets any of the following criteria, it is considered a serious adverse event (SAE): Death of patients, life-threatening, hospitalization, prolongation of hospitalization, congenital anomaly, persistent or significant disability/incapacity, important medical event requiring medical or surgical in- 
tervention to prevent serious outcome. The following definitions were used to rate the severity for any adverse event: Mild; the AE is transient and easily tolerated by the patient. Moderate; the AE causes the patient discomfort and interrupts the patient's usual activities. Severe; the $\mathrm{AE}$ causes considerable interference with the patient's usual activities and may be incapacitating or life threatening. All patients provided informed consent and the study was conducted in accordance with the protocol, International Conference on Harmonization, and the Helsinki Declaration of 1964 as revised in 2013. Instituituional Review Board (IRB) approval was obtained from all 9 sites (approval number: AJIRB-SBR-SUR-15-244; Board Name: Institution Review Board of Ajou University Hospital).

\section{Patient-reported outcomes}

PRO were recorded 3 times using the HAQ-DI, SF-36, EQ-5D-3L, and WPAI at baseline, Week 12, and Week 24.

\section{1) HAQ-DI}

The HAQ-DI is a patient-reported questionnaire that assesses functioning impacted by RA $[7,14]$. It includes the categories of dressing, grooming, arising, eating, walking, hygiene, reaching, gripping, and common daily activities. It asks patients about the amount of difficulty they experience in these activities as well as the use of aids and/or devices. The HAQ also has a numeric rating scale that assesses pain on a scale from 0 to 10 [15]. This self-administered test takes approximately $3 \sim 4$ minutes to complete. Clinically meaningful improvement on HAQ-DI is defined as a -0.22 or greater point improvement [16].

\section{2) $\mathrm{SF}-36$}

The SF-36 is a patient-reported questionnaire of patient HRQOL $[8,9]$. It measures generic health concepts relevant across age, disease, and treatment groups. There are 36 total items that cover the recall period of the previous 4 weeks. The SF- 36 consists of 8 scaled scores that are the weighted sums of the questions in their section. The SF36 measurements can be summarized into a physical component summary (PCS) score and a mental component summary (MCS) score. Each score ranges from 0 to 100, where higher scores represent better health and functioning.

\section{3) $\mathrm{EQ}-5 \mathrm{D}-3 \mathrm{~L}$}

The EQ-5D-3L measures the patient's overall health status in a descriptive system of HRQOL states consisting of five dimensions (mobility, self-care, usual activities, pain/discomfort, and anxiety/depression), each of which can take one of three responses. The responses record three levels of severity (no problems, some problems, and extreme problems) within a particular EQ-5D-3L dimension [10]. In addition, a visual analog scale rates current health status from 0 to 100 . The EQ-5D-3L results can be converted to health utility scores.

\section{4) WPAI}

The WPAI is a patient-reported questionnaire that measures work and activity impairments during the previous 7 days. It determines employment status, hours missed from work due to disease (i.e., RA), hours of work missed for other reasons, hours worked, the degree to which the disease affected work productivity while at work, and the degree to which the disease-affected activities outside of work (overall activity impairment, absenteeism, presenteeism, and activity impairment) [16,17]. Additional questions around employment status were incorporated into the survey.

Scores are calculated as impairment percentages with higher percentages indicating greater impairment and less productivity.

\section{Statistical analysis}

To investigate the effects of adalimumab on HRQOL, the change in HAQ-DI, SF-36, EQ-5D-3L, and WPAI scores measured at baseline, Weeks 12 and 24 after adalimumab administration were examined for statistical significance. A descriptive analysis was conducted of all key parameters; values are presented as mean, standard deviation (SD), minimum, maximum, and median when continuous and as "n" and percent when categorical. For categorical measures, data included frequency (number of cases [n]) and percentage (\%) of total study patients observed in each category. Changes from adalimumab initiation to Week 24 were calculated and evaluated for significant improvements using paired t-tests and analysis of variance (ANOVA) for parametric data and the Wilcoxon signed-rank test for non-parametric data. The mean changes in HAQ-DI score was tested with a paired t-test without adjusting for baseline disease severity and showed by p-value; for sensitivity analysis, the mean change was analysed using ANOVA adjusting for baseline 
disease severity (DAS28) and showed by p-value. Changes in SF-36 (PCS, MCS), EQ-5D-3L index, and WPAI at Weeks 12 and 24 after the initiation of adalimumab was tested with paired t-test without adjustment for baseline disease severity. Efficacy measures were not assessed after a patient discontinued adalimumab. The exception to this was the analysis of proportion of patients at 24 weeks who remained on adalimumab. Item level data on the PROs were imputed according to the developers' recommendations. There was no other imputation for missing data, except for dates in which the exact day was missing; in such a case, the missing day was replaced by day " 15 ". For the dates that had missing month or year, the date was treated as missing.

\section{RESULTS}

\section{Baseline characteristics of patients with RA}

The demographic characteristics of RA patients in this

Table 1. Baseline characteristics of patients

\begin{tabular}{lc}
\hline \hline \multicolumn{1}{c}{ Characteristic } & $\begin{array}{c}\text { Patients } \\
(\mathrm{n}=91)\end{array}$ \\
\hline Age (yr) & $55.7 \pm 13.3$ \\
Age group & \\
$18 \sim 34 \mathrm{yr}$ & $7(7.7)$ \\
$35 \sim 44 \mathrm{yr}$ & $9(9.9)$ \\
$45 \sim 54 \mathrm{yr}$ & $18(19.8)$ \\
$55 \sim 64 \mathrm{yr}$ & $34(37.4)$ \\
$65 \sim 74$ yr & $18(19.8)$ \\
$\geq 75$ yr & $5(5.5)$ \\
Sex, female & $64(70.3)$ \\
Disease duration* (yr) & $7.67 \pm 7.34$ \\
Comorbidities & $23(25.3)$ \\
Diabetes & $9(9.9)$ \\
Chronic pulmonary disease & $5(5.5)$ \\
Cerebrovascular disease & $4(4.4)$ \\
DAS28 & \\
DAS28 categorization & $6.1 \pm 1.0$ \\
Moderate disease activity & \\
High disease activity & $6(6.6)$ \\
Concomitant disease modifying antirheumatic drug & $28(30.8)$ \\
Methotrexate & $23(25.3)$ \\
Leflunomide & $13(14.3)$ \\
Hydroxychloroquine & $11(12.1)$ \\
Sulfasalazine & $3(3.3)$ \\
Concomitant oral steroids & $23(25.3)$ \\
\hline
\end{tabular}

Values are presented as mean \pm standard deviation or number $(\%)$. ${ }^{*}$ Disease duration $=$ Year of index visit-Year of diagnosis. ${ }^{\dagger}$ DAS28-CRP or DAS28-ESR. study are summarized in Table 1. The RA patients had a mean age of 55.7 years; the majority (37.4\%) were 55 64 years of age. Only $5.5 \%$ were elderly patients (i.e., $>75$ years old). The majority of RA patients in this study were female (70.3\%). RA patients had mean disease duration of 7.67 years. A total of $25.3 \%$ of the RA patients were suffering from comorbidities, including diabetes (9.9\%), chronic pulmonary diseases $(5.5 \%)$, and cerebrovascular diseases (4.4\%). The mean DAS28 was 6.1, with the majority (93.4\%) of patients having high disease activity. Twenty-eight patients $(30.8 \%)$ were receiving concomitant disease modifying antirheumatic drug at baseline. At baseline, 91 patients were enrolled and attended a baseline visit of which 79 patients were seen for both week 12 and week 24 . A total of 12 patients did not attend all 3 visits, 9 attended baseline and one additional visit with one patient only attending baseline visit.

\section{Change in HAQ-DI at weeks 12 and 24 after initiation of adalimumab}

The changes in HAQ-DI at weeks 12 and 24 after the initiation of adalimumab are summarized in Table 2 .

According to the PREMIER study, a prominent study on adalimumab, the mean HAQ-DI baseline score of participants was 1.5 [18], while the baseline mean score in this study was 1.29 (SD, 0.71).

The mean change from baseline HAQ-DI score was statistically significant at weeks $12(\mathrm{p}<0.0001)$ and $24(\mathrm{p}<$ 0.0001 ), with the HAQ-DI scores improving from -0.46 at week 12 to -0.67 at week 24 . Clinically meaningful improvement in HAQ-DI, defined as an improvement from baseline greater than -0.22 , was achieved in $65.8 \%$ and $77.5 \%$ patients at weeks 12 and 24 , respectively. These results suggest that adalimumab treatment significantly improved patients' performance in daily life; particularly, the change in HAQ-DI showed a greater increase at week 24 than at week 12 compared to baseline, indicating that the improvement in symptoms also increased with the continued use of adalimumab.

\section{Change in SF-36 domain scales at weeks 12 and 24 after initiation of adalimumab}

In terms of SF-36 domain scores, the changes in PCS and MCS T-scores at weeks 12 and 24 after the initiation of adalimumab are summarized in Table 2 . The mean change from baseline in PCS T-score improved from 6.56 at week 12 to 9.51 at week 24. Likewise, the mean change from baseline in MCS T-score improved from 5.26 at week 12 
Table 2. Changes in HAQ-DI, EQ-5D-3L, SF-36 at week 12 and 24 after initiation of adalimumab

\begin{tabular}{|c|c|c|c|c|}
\hline \multirow{2}{*}{ Instrument } & \multirow{2}{*}{ Value } & \multicolumn{2}{|c|}{ Change from baseline } & \multirow{2}{*}{ p-value } \\
\hline & & Mean \pm SD & $95 \% \mathrm{Cl}$ & \\
\hline \multicolumn{5}{|l|}{ HAQ-DI } \\
\hline Baseline & $1.29 \pm 0.71$ & & & \\
\hline Week 12 & $0.80 \pm 0.64$ & $-0.46 \pm 0.60$ & $-0.60,-0.33$ & $<0.0001^{*}$ \\
\hline Week 24 & $0.63 \pm 0.56$ & $-0.67 \pm 0.67$ & $-0.83,-0.51$ & $<0.0001^{*}$ \\
\hline $\begin{array}{l}\text { Patients achieving a clinically meaningful } \\
\text { improvement in HAQ-DI at week } 12^{\dagger}\end{array}$ & $52(65.8)$ & & & \\
\hline $\begin{array}{l}\text { Patients achieving a clinically meaningful } \\
\text { improvement on HAQ-DI at } 24 \text { weeks }^{\dagger}\end{array}$ & $55(77.5)$ & & & \\
\hline \multicolumn{5}{|l|}{ EQ-5D-3L } \\
\hline Baseline & $0.59 \pm 0.22$ & & & \\
\hline Week 12 & $0.74 \pm 0.18$ & $0.15 \pm 0.24$ & $0.10,0.20$ & $<0.0001^{\ddagger}$ \\
\hline Week 24 & $0.77 \pm 0.19$ & $0.18 \pm 0.25$ & $0.12,0.24$ & $<0.0001^{\ddagger}$ \\
\hline \multicolumn{5}{|l|}{ SF-36 Physical component summary scores } \\
\hline Baseline & $34.57 \pm 7.76$ & & & \\
\hline Week 12 & $41.26 \pm 7.56$ & $6.56 \pm 6.98$ & $7.67,11.35$ & $<0.0001^{\ddagger}$ \\
\hline Week 24 & $43.91 \pm 7.07$ & $9.51 \pm 7.77$ & $7.67,11.35$ & $<0.0001^{\ddagger}$ \\
\hline \multicolumn{5}{|l|}{ SF-36 Mental component summary scores } \\
\hline Baseline & $35.88 \pm 11.51$ & & & \\
\hline Week 12 & $41.54 \pm 11.84$ & $5.26 \pm 11.62$ & $2.66,7.87$ & $0.0001^{\ddagger}$ \\
\hline Week 24 & $43.00 \pm 11.62$ & $6.63 \pm 12.31$ & $3.71,9.54$ & $<0.0001^{\ddagger}$ \\
\hline
\end{tabular}

Values are presented as mean \pm standard deviation or number (\%). HAQ-DI: Health Assessment Questionnaire Disability Index, EQ-5D-3L: EuroQol 5-dimension 3-Level, SF-36: Short Form 36-Item Health Survey, SD: standard deviation, Cl: confidence interval. ${ }^{*}$ p-value is from the test of mean change using ANOVA adjusting for baseline disease severity. ${ }^{\dagger}$ Clinically meaningful improvement on HAQ-DI is defined as a -0.22 or greater point improvement. ${ }^{\ddagger} p$-value is from the paired t-test testing the mean change.

Table 3. Change in WPAl at weeks 12 and 24 after initiation of adalimumab

\begin{tabular}{|c|c|c|c|c|}
\hline \multirow{2}{*}{ Instrument } & \multirow{2}{*}{ Mean \pm SD } & \multicolumn{2}{|c|}{ Change from baseline } & \multirow{2}{*}{ p-value* } \\
\hline & & Mean \pm SD & $95 \% \mathrm{Cl}$ & \\
\hline \multicolumn{5}{|c|}{ Percent overall work impairment (\%) } \\
\hline Baseline & $58.27 \pm 26.19$ & & & \\
\hline Week 12 & $37.03 \pm 26.27$ & $-19 \pm 32$ & $-32,-5$ & 0.0098 \\
\hline Week 24 & $32.31 \pm 25.86$ & $-25 \pm 31$ & $-40,-11$ & 0.0015 \\
\hline \multicolumn{5}{|c|}{ Percent activity impairment (\%) } \\
\hline Baseline & $61.71 \pm 24.98$ & & & \\
\hline Week 12 & $45.47 \pm 27.52$ & $-15 \pm 31$ & $-22,-8$ & $<0.0001$ \\
\hline Week 24 & $38.59 \pm 28.35$ & $-25 \pm 34$ & $-33,-16$ & $<0.0001$ \\
\hline
\end{tabular}

WPAI: Work Productivity and Activity Impairment, SD: standard deviation, Cl: confidence interval. *p-value is from the paired t-test testing the mean change.

to 6.63 at week 24 . These results show that adalimumab treatment significantly improved the physical and mental aspects at weeks 12 and 24 playing a significant role in improving patient HRQOL.

\section{Change in EQ-5D-3L index at weeks 12 and 24 after initiation of adalimumab}

The changes in EQ-5D-3L index at weeks 12 and 24 after the initiation of adalimumab are summarized in Table 2. EQ-5D-3L consists of 5 dimensions, including mobility, self-care, usual activities, pain/discomfort, and anxiety/ 
depression. In this study, the mean baseline utility value increased from 0.59 (SD, 0.22) at baseline to 0.74 (SD, 0.18 ) at week 12 and 0.77 (SD, 0.19) at week 24. A statistically significant mean change from baseline in EQ5D-3L index score was observed at week $12(0.15 ; \mathrm{p}<$ $0.0001)$ as well as week $24(0.18 ; \mathrm{p}<0.0001)$. In the EQ-5D-3L index, the higher the score, the greater the improvement in HRQOL - an increase of 0.18 in the mean score at week 24 after the use of adalimumab indicated improved HRQOL.

\section{Change in WPAI at weeks 12 and 24 after initiation of adalimumab}

The changes in WPAI index at weeks 12 and 24 after the initiation of adalimumab are summarized in Table 3. Significant mean changes in percentage overall work impairment of -0.19 and -0.25 were observed at weeks 12 and 24 , respectively $(\mathrm{p} \leq 0.01)$. The change from baseline in percentage of activity impairment was -0.15 at week 12 and -0.25 at week 24 . This indicates that patients with active RA showed clinically significant improvement in occupational and daily activities at weeks 12 and 24 after adalimumab treatment.

\section{Adverse events and serious adverse events}

There were $10 \mathrm{AE}$ reported in 6 patients (6.6\%) during

Table 4. Cumulative incidence of non-serious adverse events and serious adverse events

\begin{tabular}{lc}
\hline \multicolumn{1}{c}{ Adverse events } & $\begin{array}{c}\text { Cumulative } \\
\text { incidence (\%) }\end{array}$ \\
\hline Non-serious adverse events & 2.27 \\
Rash & 2.27 \\
Injection site erythema & 2.27 \\
Injection site pruritus & 1.14 \\
Injection site rash & 1.14 \\
Increased antinuclear antibody & 1.14 \\
Increased CRP & 1.14 \\
Diabetes mellitus & 1.14 \\
Increased RBC sedimentation rate & 1.14 \\
Decreased hemoglobin & 1.14 \\
Rosacea & \\
Serious adverse events & 1.14 \\
Deep vein thrombosis & 1.14 \\
Diabetes mellitus & 1.14 \\
Intervertebral disc protrusion & 1.14 \\
Pneumonia bacterial & 1.14 \\
Spinal column stenosis &
\end{tabular}

CRP: C-reactive protein, RBC: red blood cell. the study (Table 4). Six patients experienced AE including rash, rash on injection site, rosacea. Serious adverse events were reported in 6 patients $(6.6 \%, 5$ events). Six patients experienced serious adverse events including deep vein thrombosis, herniated lumbar disc, spinal stenosis, and bacterial pneumonia.

\section{DISCUSSION}

Patients with RA suffer from both joint pain due to chronic inflammation and difficulties in daily activities such as dysfunction, depression, and work performance. PRO reflects the life status of these patients including HRQOL, physical function, disability, fatigue, sleep, mental health status, work productivity, and work activity impairment [18]. This information obtained through direct communication with the patient can be used to better understand the patient's mood or functional status and can be applied to therapy [19]. Many clinical studies have used PRO as a tool to assess drug efficacy. Kievit et al. [20] compared the efficacies of etanercept, infliximab, and adalimumab in 707 RA patients and reported through SF-36 PCS score, DAS28 score, and HAQ-DI data that the effects of infliximab did not match those of etanercept and adalimumab. Adalimumab has been used in Korea for more than 15 years, but no studies have evaluated its effect on Korean patient HRQOL. In this study, significant improvement in quality of life indicators was observed at weeks 12 and 24 in the Korean patients with high disease activity who did not respond to conventional medication. In particular, a change of -0.22 or more in HAQ-DI indicates a clinically significant improvement [16], and $65 \%$ and $77.5 \%$ of patients in this study showed significant improvements in quality of life at weeks 12 and 24. According to the PREMIER study, which investigated the effects of adalimumab by comparing HAQ-DI and SF-36 PCS scores, among the methotrexate (MTX) monotherapy group, adalimumab monotherapy group, and MTX plus adalimumab group, the greatest improvement was seen in the MTX plus adalimumab group at 2 years compared with baseline [21]. The SF-36 PCS score showed a mean increase of 14 points at weeks 13 and 26 in the 12-week MTX plus adalimumab group, while the adalimumab monotherapy group showed an increase of about 9 points at week 12 and 10 points at week 26 . In this study, the mean change in PCS score at week 12 after adalimumab administration was 6.56 , while that at week 24 was 9.51 . This is similar to the 16 -week outcome ob- 
served with the combined treatment of etanercept and MTX in Asian patients with RA in 2013 [22].

The EQ-5D-3L is a simple and well-established index that reflects patient-oriented status [10]. The highest score is expressed as 1.0, and the higher the score, the greater the improvement in quality of life. In a study conducted in Sweden in 2017 that analyzed drug effects using the EQ-5D-3L indices after single use of various biological agents, the change after 6 months of using infliximab and rituximab was 0.11 , while the mean change in the score of 8 drugs including golimumab, certolizumab, tocilizumab, etanercept, abatacept, and adalimumab was 0.23 [23]. In the present study of Korean patients, the EQ-5D-3L score increased by 0.18 after 6 months, which is a significant change in quality of life. The mean disease duration for the patients participating in this study was 7.67 years: During this period, pain or disability interfered with their activities and they had difficulty performing their work. Previously published studies showed that patients with long-term illnesses are more likely to experience difficulties with employment [24] than those with early-stage RA. This suggests that proper management of RA as a chronic disease is required. In addition, a study of Japanese patients reported that $67 \%$ experienced work and activity impairments, stating that the promotion of active remission is required to overcome this phenomenon [25]. The WPAI is a key indicator of job performance. A significant improvement in overall work and activity impairments was observed in a US study examining the change in WPAI after 6 months of treatment with etanercept in patients with moderate or severe disease activity [26]. Similarly, a study conducted in the Middle East reported that the use of adalimumab for 6 months improved quality of life, reduced fatigue, and increased productivity in patients with RA [27].

The mean age of patients with active RA participating in this study was 55.7 years; considering that they are in the age group that can still be actively working, proper treatment with adalimumab is expected to reduce the loss of labor capacity and be of great help in terms of social activities by cutting economic losses for the patients.

Using objective indices such as the HAQ-DI, SF-36, and EQ-5D-3L, this study revealed that the quality of life of patients with RA in Korea could be significantly improved using adalimumab. It was demonstrated through multicenter studies in Korea that this improvement in quality of life also reduced labor force losses and helped patients play a social role. In particular, this study is valuable as the first of its kind to verify the therapeutic effect of adalimumab in patients with RA with moderate or severe disease activity in Korea using the quality of life index.

There is a limitation that use of drugs, economic status of patients have not been adjusted and that comparison with control group were not performed. Unlike clinical trials, obtainable data are limited and may be missing. In addition, sample size could be improved, and we believe that stable results can be obtained with a longer observational period.

\section{CONCLUSION}

The results of the present study in Korea demonstrate that adalimumab produced clinically important and statistically significant improvement in health-related quality of life outcomes at weeks 12 and 24 as validated using different PRO instruments. Adalimumab can improve the quality of life of RA patients who suffered from pain, disability, fatigue, and depression.

\section{ACKNOWLEDGMENTS}

The design, study conduct, and financial support for the study were provided by AbbVie (Grant number: P15-777). AbbVie participated in the interpretation of data, writing, review, and approval of the publication.

\section{CONFLICT OF INTEREST}

Dr. Yoon-Kyoung Sung has received research funding or speaker fees from BMS, Eisai, JW pharmaceuticals, Pfizer and Yuhan. Yusun Lee is an employee of Abbvie. Dr. Myeoung-su Lee, Chang Hoon Lee, Hye Soon Lee, Jung Ran Choi, Kyungsu Park, Mi-Kyoung Lim, Byoong Yong Choi, Hyoun-Ah Kim, Seung Won Choi, and Wan-Hee Yoo have no conflict of interest.

\section{AUTHOR CONTRIBUTIONS}

M.L., C.H.L., H.S.L., Y.K.S., J.R.C., K.P., M.K.L., B.Y.C., H.A.K., S.W.C., Y.L. and W.H.Y. contributed to the study concept and design. Material preparation, data collection and analysis were performed by M.L. and W.H.Y. The first draft of the manuscript was written by M.L. and all authors commented on previous versions of the manuscript. All authors read and approved the final manuscript. 


\section{REFERENCES}

1. Silman AJ, Pearson JE. Epidemiology and genetics of rheumatoid arthritis. Arthritis Res 2002;4 Suppl 3:S265-72.

2. Hyrich K, Symmons D, Watson K, Silman A; BSRBR Control Centre Consortium; British Society for Rheumatology Biologics Register. Baseline comorbidity levels in biologic and standard DMARD treated patients with rheumatoid arthritis: results from a national patient register. Ann Rheum Dis 2006;65:895-8.

3. Dougados M, Soubrier M, Antunez A, Balint P, Balsa A, Buch MH, et al. Prevalence of comorbidities in rheumatoid arthritis and evaluation of their monitoring: results of an international, cross-sectional study (COMORA). Ann Rheum Dis 2014;73:62-8.

4. Choi IA, Lee JS, Song YW, Lee EY. Mortality, disability, and healthcare expenditure of patients with seropositive rheumatoid arthritis in Korea: a nationwide population-based study. PLoS One 2019;14:e0210471.

5. Uhlig T, Loge JH, Kristiansen IS, Kvien TK. Quantification of reduced health-related quality of life in patients with rheumatoid arthritis compared to the general population. J Rheumatol 2007;34:1241-7.

6. Matcham F, Scott IC, Rayner L, Hotopf M, Kingsley GH, Norton $S$, et al. The impact of rheumatoid arthritis on quality-of-life assessed using the SF-36: a systematic review and meta-analysis. Semin Arthritis Rheum 2014;44:123-30.

7. Bruce B, Fries JF. The Stanford health assessment questionnaire: a review of its history, issues, progress, and documentation. J Rheumatol 2003;30:167-78.

8. Ware JE Jr, Sherbourne CD. The MOS 36-item short-form health survey (SF-36). I. conceptual framework and item selection. Med Care 1992;30:473-83.

9. Ware JE Jr, Gandek B. Overview of the SF-36 health survey and the international quality of life assessment (IQOLA) project. J Clin Epidemiol 1998;51:903-12.

10. EuroQol Group. EuroQol--a new facility for the measurement of health-related quality of life. Health Policy 1990;16: 199-208.

11. Atzinger CB, Guo JJ. Biologic disease-modifying antirheumatic drugs in a national, privately insured population: utilization, expenditures, and price trends. Am Health Drug Benefits 2017;10:27-36.

12. Bang LM, Keating GM. Adalimumab: a review of its use in rheumatoid arthritis. BioDrugs 2004;18:121-39.

13. Center for Drug Evaluation and Research, Center for Biologics Evaluation and Research. Application number: 125057/0. Approval letter(s) [Internet]. Rockville (MD): Food and Drug Administration, c2002 [cited 2019 Jun 25]. Available from: https://www.accessdata.fda.gov/drugsatfda_docs/nda/2002/BLA_125057_S000_HUMIRA_ APPROV.PDF.

14. Fries JF, Spitz P, Kraines RG, Holman HR. Measurement of patient outcome in arthritis. Arthritis Rheum 1980;23: 137-45.

15. Hawker GA, Mian S, Kendzerska T, French M. Measures of adult pain: visual analog scale for pain (VAS Pain), numeric rating scale for pain (NRS Pain), McGill pain questionnaire (MPQ), short-form McGill pain questionnaire (SF-MPQ), chronic pain grade scale (CPGS), short form-36 bodily pain scale (SF-36 BPS), and measure of intermittent and constant osteoarthritis pain (ICOAP). Arthritis Care Res (Hoboken) 2011;63 Suppl 11:S240-52.

16. Wells GA, Tugwell P, Kraag GR, Baker PR, Groh J, Redelmeier DA. Minimum important difference between patients with rheumatoid arthritis: the patient's perspective. J Rheumatol 1993;20:557-60.

17. Reilly M. Work productivity and activity impairment questionnaire: low back pain (WPAI-LBP) [Internet]. New York (NY): Reilly Associates, c2002 [cited 2019 Jun 25]. Available from: http://www.reillyassociates.net/English_ US_WPAI-LBP_V2.pdf.

18. Kekow J, Moots RJ, Emery P, Durez P, Koenig A, Singh A, et al. Patient-reported outcomes improve with etanercept plus methotrexate in active early rheumatoid arthritis and the improvement is strongly associated with remission: the COMET trial. Ann Rheum Dis 2010;69:222-5.

19. Gossec L, Dougados M, Dixon W. Patient-reported outcomes as end points in clinical trials in rheumatoid arthritis. RMD Open 2015;1:e000019.

20. Kievit W, Adang EM, Fransen J, Kuper HH, van de Laar MA, Jansen TL, et al. The effectiveness and medication costs of three anti-tumour necrosis factor alpha agents in the treatment of rheumatoid arthritis from prospective clinical practice data. Ann Rheum Dis 2008;67:1229-34.

21. Breedveld FC, Weisman MH, Kavanaugh AF, Cohen SB, Pavelka K, van Vollenhoven R, et al. The PREMIER study: a multicenter, randomized, double-blind clinical trial of combination therapy with adalimumab plus methotrexate versus methotrexate alone or adalimumab alone in patients with early, aggressive rheumatoid arthritis who had not had previous methotrexate treatment. Arthritis Rheum 2006; 54:26-37.

22. Bae SC, Gun SC, Mok CC, Khandker R, Nab HW, Koenig AS, et al. Improved health outcomes with etanercept versus usual DMARD therapy in an Asian population with established rheumatoid arthritis. BMC Musculoskelet Disord 2013;14:13.

23. Jørgensen TS, Turesson C, Kapetanovic M, Englund M, Turkiewicz A, Christensen R, et al. EQ-5D utility, response and drug survival in rheumatoid arthritis patients on biologic monotherapy: a prospective observational study of patients registered in the south Swedish SSATG registry. PLoS One 2017;12:e0169946.

24. Han C, Smolen J, Kavanaugh A, St Clair EW, Baker D, Bala M. Comparison of employability outcomes among patients with early or long-standing rheumatoid arthritis. Arthritis Rheum 2008;59:510-4.

25. Kim D, Kaneko Y, Takeuchi T. Importance of obtaining remission for work productivity and activity of patients with rheumatoid arthritis. J Rheumatol 2017;44:1112-7.

26. Hone D, Cheng A, Watson C, Huang B, Bitman B, Huang $\mathrm{XY}$, et al. Impact of etanercept on work and activity impairment in employed moderate to severe rheumatoid arthritis patients in the United States. Arthritis Care Res (Hoboken) 2013;65:1564-72.

27. Hussain W, Janoudi N, Noorwali A, Omran N, Baamer M, Assiry el $\mathrm{H}$, et al. Effect of adalimumab on work ability assessed in rheumatoid arthritis disease patients in Saudi Arabia (AWARDS). Open Rheumatol J 2015;9:46-50. 